\title{
ARTICLE
}

Received 16 Oct 2012 | Accepted 7 Feb 2013 | Published 12 Mar $2013 \quad$ DOl: 10.1038/ncomms2583

\section{Epitaxial lift-off process for gallium arsenide substrate reuse and flexible electronics}

Cheng-Wei Cheng ${ }^{1}$, Kuen-Ting Shiu ${ }^{1}$, Ning Li ${ }^{1}$, Shu-Jen Han ${ }^{1}$, Leathen Shi ${ }^{1} \&$ Devendra K. Sadana ${ }^{1}$

Epitaxial lift-off process enables the separation of III-V device layers from gallium arsenide substrates and has been extensively explored to avoid the high cost of III-V devices by reusing the substrates. Conventional epitaxial lift-off processes require several post-processing steps to restore the substrate to an epi-ready condition. Here we present an epitaxial liftoff scheme that minimizes the amount of post-etching residues and keeps the surface smooth, leading to direct reuse of the gallium arsenide substrate. The successful direct substrate reuse is confirmed by the performance comparison of solar cells grown on the original and the reused substrates. Following the features of our epitaxial lift-off process, a high-throughput technique called surface tension-assisted epitaxial lift-off was developed. In addition to showing full wafer gallium arsenide thin film transfer onto both rigid and flexible substrates, we also demonstrate devices, including light-emitting diode and metal-oxidesemiconductor capacitor, first built on thin active layers and then transferred to secondary substrates.

\footnotetext{
${ }^{1}$ IBM T. J. Watson Research Center, 1101 Kitchawan Road, Yorktown Heights, New York 10598, USA. Correspondence and requests for materials should be addressed to C.-W.C. (email: chengwei@us.ibm.com).
} 
G allium arsenide (GaAs), with its high electron mobility and direct bandgap, has been employed in high performance RF electronics and optoelectronics for decades $^{1-4}$. On the basis of thermodynamics calculation, its bandgap lies at the energy for the theoretical maximum efficiency of single junction (SJ) solar cells and makes it an ideal material for high efficiency solar cells $s^{5}$. However, the high cost of GaAs substrates hinders them from being widely adopted in certain applications, in particular for solar cell because of a need for large amounts of the material. A typical thickness of a GaAs substrate is around a few hundred micrometres, and only a few micrometres or nanometres thickness is required to fully absorb the solar energy or serve as the active layer for device operation. Therefore, the material cost can be potentially reduced if thin device layers can be separated from the substrate while keeping the substrate intact and ready for another epitaxial growth.

In 1978, Konagai et al. ${ }^{6}$ proposed a method which is known today as epitaxial lift-off (ELO) to separate a device layer from a GaAs substrate by using hydrofluoric acid (HF) to selectively etch a AlGaAs (aluminium compound) sacrificial layer inserted between the device film and the substrate (Fig. 1(a)). Subsequently, this same method has been applied by many researchers to successfully peel GaAs thin films from the parent substrates and transfer them onto desirable substrates for various applications ${ }^{7,8}$. The major drawback of this method arises from the high surface roughness of the parent substrate as well as reaction residues left on the substrate after the ELO process, and post-process steps, for example, chemicalmechanical-polish or chemical etching, typically required to reclaim the substrate ${ }^{9}$. In addition, $\mathrm{HF}$ is a lethal and highly corrosive acid requiring special handling and extra protection. Compared with typical short processing times with HF in silicon industry, the conventional ELO process takes hours to complete, which potentially increases the exposure risk for operators and the operating cost. Hence, developing a new ELO process with different chemistry that enables the direct reuse of the substrate is very desirable to lower the overall cost. Although HCl-based etchant was proposed and used by several researchers to selectively etch $\mathrm{AlGaAs}{ }^{10,11}$ or other sacrificial layers ${ }^{12-14}$, the possibility of reusing the substrate directly with these methods has not been explored. In this paper, we demonstrate a new ELO process where aluminium-arsenide based sacrificial layer and HF-based etchant are replaced with phosphide-based materials and $\mathrm{HCl}$. This new approach minimizes the amount of postetching residues and provides the surface passivation that keeps the surface smooth during ELO process, leading to direct reuse of GaAs substrate.

\section{Results}

Mechanism of the ELO Process for reuse of GaAs substrates. A viable ELO process for manufacturing should satisfy three essential conditions: (1) the surface of the reused substrate needs to be residue-free, and have low surface roughness (the rootmean-square (RMS) roughness of an epi-ready GaAs wafer is about $0.3 \mathrm{~nm}$ ); (2) the properties of subsequent layers grown on reused substrates should be identical to that of the first released film; (3) the throughput of the ELO process should be high to drive down overall cost. Fig. 1(b) illustrates the chemical reactions of the conventional ELO process and atomic-force microscopy (AFM) image of the post-ELO substrate surface. The attack of the substrate by the HF and the residues formed during the sacrificial layer etching result in the increase of RMS roughness from 0.3 to $1-4 \mathrm{nms}$ range. Chemical reactions between AlAs and HF have been well studied ${ }^{10,15,16}$ and the two major reactions are shown below:

$$
\begin{aligned}
& \operatorname{AlAs}_{(\mathrm{s})}+3 \mathrm{HF}_{(\mathrm{aq})} \rightarrow \mathrm{AlF}_{3(\mathrm{~s})}+\mathrm{AsH}_{3(\mathrm{~g})} \\
\mathrm{AlAs}_{(\mathrm{s})}+ & 3 \mathrm{HF}_{(\mathrm{aq})}+6 \mathrm{H}_{2} \mathrm{O} \rightarrow \mathrm{AsH}_{3(\mathrm{~g})}+\left[\mathrm{AlF}_{n}\left(\mathrm{H}_{2} \mathrm{O}\right)_{6-n}\right]_{(\mathrm{s})}^{(3-n)+} \\
+ & (3-n) \mathrm{F}_{(\mathrm{aq})}^{-}+n \mathrm{H}_{2} \mathrm{O}
\end{aligned}
$$

Three primary byproducts from these etching reactions are $\mathrm{AlF}_{3},\left[\mathrm{AlF}_{n}\left(\mathrm{H}_{2} \mathrm{O}\right)^{6-n}\right](3-n)+$ and $\mathrm{AsH}_{3} . \mathrm{AsH}_{3}$ is gaseous and can form bubbles and diffuse away from the interface to the atmosphere. $\mathrm{AlF}_{3}$ and $\left[\mathrm{AlF}_{n}\left(\mathrm{H}_{2} \mathrm{O}\right)^{6-n}\right](3-n)+$, on the other hand, are solid and hard to dissolve into the solution. Besides these primary byproducts, solid $\mathrm{As}_{2} \mathrm{O}_{3}$ can also be generated on the substrate depending on the oxygen concentration of the etchant ${ }^{17}$. Table 1 summarizes the solubility of the solid byproducts from the conventional ELO process ${ }^{18}$. Although $\mathrm{AlF}_{3}$ and $\mathrm{As}_{2} \mathrm{O}_{3}$ do not have extremely low aqueous solubilities, it is evident that they still remain on the substrate surface after ELO and cause a high surface roughness, which can be seen in Fig. 1(b). Moreover, HF used in this process slowly attacks GaAs and induces additional surface roughness. Post-chemical etching with thermal cleaning can potentially reduce the surface roughness to the level of the original wafer. However, the remaining contaminants still seriously degrade the quality of regrown films and device performances 9 . To completely remove all these contaminants and achieve high quality regrown films, multi-step chemical polishing with different sacrificial (protection) layers was recently proposed, with the penalties of the higher cost for extra epitaxial growths and HF still being used as the main etchant ${ }^{19}$.

The abovementioned issues in the conventional ELO process can be mitigated by employing a different sacrificial layer that can be selectively etched by a non-HF solution, while leaving no insoluble etching byproducts on the GaAs surface. One advantage of compound semiconductors is that their physical and chemical properties can be manipulated by alloying different group III and V elements. For example, phosphidebased materials (InGaP, InAlP, InP and so on) have been widely applied as etch stop layers for the selective etching of arsenide based materials (GaAs, InGaAs and so on), and vice versa. It is well known that hydrochloric acid $(\mathrm{HCl})$ can etch phosphidebased materials ${ }^{14,20-22}$ and the reaction is shown in Equation (3):

$$
\begin{gathered}
\mathrm{InXP}_{(\mathrm{s})}+\mathrm{HCl}_{(\mathrm{aq})} \rightarrow \operatorname{lnCl}_{3(\mathrm{aq})}+\mathrm{XCl}_{3(\mathrm{aq})}+\mathrm{PH}_{3(\mathrm{~g})} \\
X=\mathrm{Al}, \mathrm{Ga}
\end{gathered}
$$

The gaseous $\mathrm{PH}_{3}$ diffuses away from the interface and the rest of the etching byproducts are highly soluble (their solubilities are shown in Table 1); thus, no residues are left on the wafer surface. Fig. 1(c) illustrates the new ELO process and AFM image of the post-ELO surface. The wafer surface is atomically flat with RMS roughness of merely $0.349 \mathrm{~nm}$-significantly improved over conventional ELO (Supplementary Fig. S1). This improvement comes from the almost infinite etch-selectivity between $\mathrm{GaAs}$ and phosphide with the $\mathrm{HCl}$ etchant. In order to verify that $\mathrm{HCl}$ attacks $\mathrm{GaAs}$ much less compared with $\mathrm{HF}$, we soaked blanket $\mathrm{GaAs}$ wafers in $\mathrm{HCl}$ or $\mathrm{HF}$ for $24 \mathrm{~h}$ and the AFM images afterwards are shown in Fig. 2(a). RMS roughness of the $24 \mathrm{~h}-\mathrm{HCl}$ wafer is only $0.327 \mathrm{~nm}$, much lower than the $1.666 \mathrm{~nm}$ RMS roughness after $24 \mathrm{~h}$ in HF. This result indicates that HF attacks GaAs severely and causes excess surface roughness even without the ELO process. This can be explained through 
a
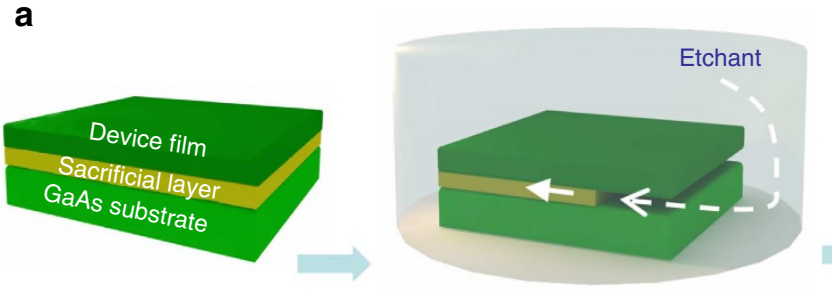

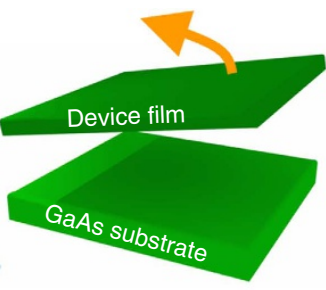

HF $49 \%$

RMS $2.955 \pm 0.214 \mathrm{~nm}$

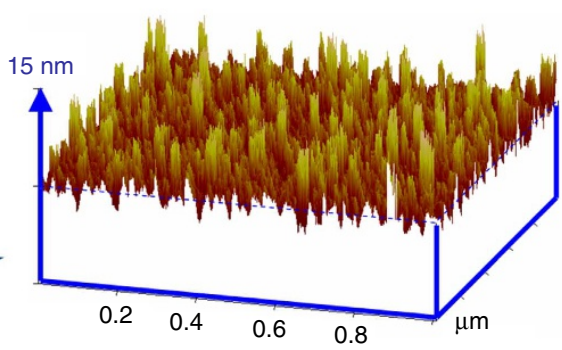

$\mathrm{HCl} 36 \%$

RMS $0.349 \pm 0.031 \mathrm{~nm}$

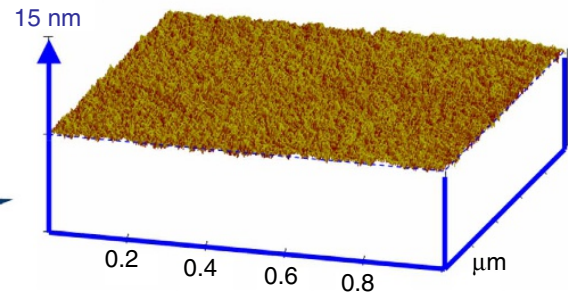

Figure 1 | Concept of epitaxial lift-off (ELO) process and post-ELO GaAs surface morphologies with conventional and novel ELO processes.

(a) Schematic illustration of general ELO process. (b,c) Schematic illustrations of the chemical reactions near the sacrificial layer/etchant interfaces during the conventional and the novel ELO process and the three-dimensional AFM images of the GaAs surfaces after the processes. Here, indium aluminium phosphide (InAIP) was taken as an example of the sacrificial layer of the novel ELO process.

Table 1 | Solubility of etching products from conventional and novel ELO process.

Solubility of etching products

Conventional ELO

Compound mole per $100 \mathrm{~g} \mathrm{H}_{2} \mathrm{O}$

$\mathrm{AlF}_{3}$

$\mathrm{Al}(\mathrm{OH})_{3}$

$6.71 \times 10^{-3}$

$1.28 \times 10^{-6}$

$\mathrm{As}_{2} \mathrm{O}_{3}$

$1.04 \times 10^{-2}$

$\mathrm{As}_{2} \mathrm{O}_{5}$

$2.97 \times 10^{-14}$

$\star \mathrm{NaCl}$ as a reference for solubility $\rightarrow$

$\begin{array}{cl}\text { per } 100 \mathrm{~g} \mathrm{H}_{2} \mathrm{O} & \text { Compound } \\ 0.5 & \mathrm{InCl}_{3} \\ 9.47 \times 10^{-5} & \mathrm{AlCl}_{3} \\ 2.06 & \mathrm{GaCl}_{3} \\ 4.6 \times 10^{-12} & \\ & \mathrm{NaCl}\end{array}$

\section{Novel ELO}

mole per $100 \mathrm{~g} \mathrm{H}_{2} \mathrm{O}$

0.88

0.52

Very soluble

0.615

36.0

Abbreviation: ELO, epitaxial lift-off.

$\mathrm{NaCl}$ is taken as the reference of solubility ${ }^{18}$

possible etching mechanisms for $\mathrm{GaAs}$ with $\mathrm{HF}$ and $\mathrm{HCl}$, as shown in Equation (4). In contrast to the etching of GaAs by HF that is thermodynamically favourable $(\Delta G<0)$, the reaction with $\mathrm{HCl}$ is suppressed $(\Delta \mathrm{G}>0)^{18}$.

$$
\begin{gathered}
\mathrm{GaAs}_{(\mathrm{s})}+3 \mathrm{HX}_{(\mathrm{aq})} \rightarrow \mathrm{GaX}_{3(\mathrm{aq})}+\mathrm{AsH}_{3(\mathrm{~g})} \quad X=\mathrm{F}, \mathrm{Cl} \\
\Delta \mathrm{G}_{(\mathrm{HF})}=-112.2(\mathrm{~kJ} / \mathrm{mole}) ; \\
\Delta G_{(\mathrm{HCl})}=+75.5(\mathrm{~kJ} / \mathrm{mole})
\end{gathered}
$$

Therefore, the etch rate of $\mathrm{GaAs}$ in $\mathrm{HCl}$ is much lower and the surface remains smoother. Note that in the particular alloy example in Equation (3), aluminium is not required for selective etching, but it can accelerate etching of the sacrificial layer. Moreover, because only undissociated $\mathrm{HCl}$ molecules interact with phosphide, the throughput is greatly enhanced by using concentrated $\mathrm{HCl}$ with the new ELO process ${ }^{20,23,24}$. The use of $\mathrm{HCl}$ as an etchant also poses significantly lower risks compared with highly lethal and corrosive HF. These attributes makes the new ELO process much safer and economical for manufacturing compared with the conventional method.

In addition to reducing substrate etching to minimize surface roughness, surface passivation of GaAs by acids also has an important role. Fig. 2(a) shows that GaAs wafers soaked in diluted acids display higher RMS roughness than those soaked in concentrated acids. It is well known that the combination of 
a

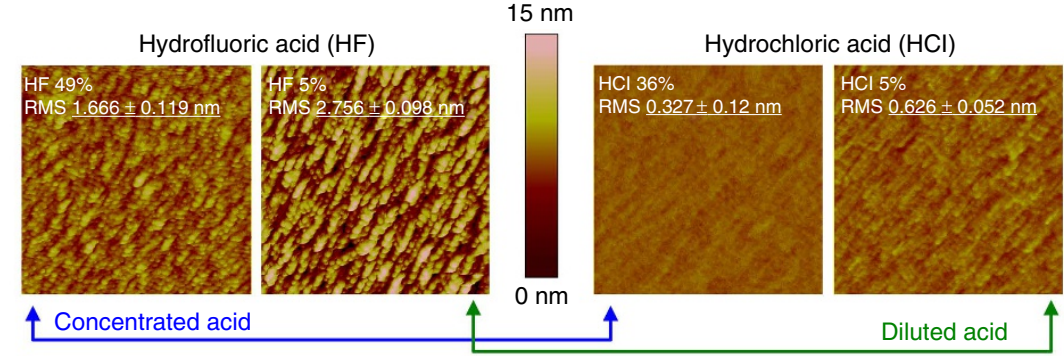

b
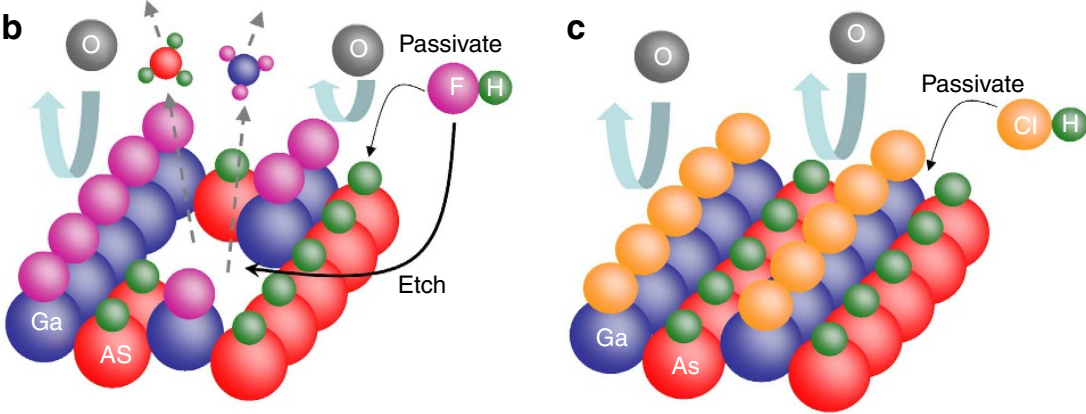

Figure 2 | Surface morphologies of GaAs surfaces during ELO process. (a) AFM images of the GaAs substrate surface dipped in both concentrated and diluted $\mathrm{HF}$ and $\mathrm{HCl}$ for 1 day. $(\mathbf{b}, \mathbf{c})$ are the schematic illustrations of the surface chemistry of $\mathrm{GaAs}$ dipped in $\mathrm{HF}$ and $\mathrm{HCl}$, respectively.

acid or base with oxidants can be an effective GaAs etchant and dissolved oxygen can serve as an oxidant. On the other hand, both $\mathrm{HF}$ and $\mathrm{HCl}$ can passivate the surface and prevent it from being oxidized by dissolved oxygen (Supplementary Fig. S2 and Supplementary Methods). The competition between surface passivation by acids and etching by dissolved oxygen determines the surface roughness at different acid concentrations. These processes are illustrated in Fig. 2(b,c). It has been proven that the GaAs surface becomes highly hydrophobic (like $\mathrm{H}$-terminated surface that forms by dipping Si into HF) after it is dipped into $\mathrm{HF}$ or $\mathrm{HCl}^{25,26}$. The surface gallium atoms are passivated by fluorine or chlorine atoms and the surface arsenic atoms are possibly passivated by hydrogen atoms ${ }^{25,27-29}$. Higher acid concentrations provide better coverage of passivated atoms and leave fewer unprotected sites to be oxidized by dissolved oxygen. The main difference between $\mathrm{HCl}$ and $\mathrm{HF}$ is that $\mathrm{HCl}$ only passivates the surface while $\mathrm{HF}$ not only passivates the surface, but slowly etches the bulk GaAs. In order to verify the hypotheses discussed above, a GaAs sample was placed into diluted (5\%) $\mathrm{HCl}$ purged with pure $\mathrm{N}_{2}$ to release dissolved oxygen from the solution for 2 days(Supplementary Fig. S3 and Supplementary Methods). The final surface roughness is $0.358 \mathrm{~nm}$ and is almost identical to the GaAs sample soaked in concentrated $\mathrm{HCl}$ for 1 day, suggesting that dissolved oxygen in diluted $\mathrm{HCl}$ roughens the surface. To quantify the etch rates of GaAs with $\mathrm{HCl}$ and HF, GaAs samples were left in $36 \% \mathrm{HCl}, 5 \% \mathrm{HF}$ and $49 \% \mathrm{HF}$ for 9 days. The etch depths were measured to be 3,12 and $100 \mathrm{~nm}$, respectively, (Supplementary Fig. S4 and Supplementary Methods). We attribute the minor etching of $36 \% \mathrm{HCl}$ to dissolved oxygen in $\mathrm{HCl}$ and confirm the significantly higher $\mathrm{GaAs}$ etch rate in $\mathrm{HF}$.

Demonstration of substrate reuse. As a smooth surface does not guarantee re-usability of the wafer after $\mathrm{ELO}^{9}$, SJ solar cell structures were grown and fabricated on both the original substrate and the reused substrate to confirm the quality of the regrown layers. Figure 3(a) shows current density versus voltage $(\mathrm{J}-\mathrm{V})$ characteristics measured under AM1.5 sun condition, and
Fig. 3(b) shows the external quantum efficiencies (EQE) of both cells. No substantial difference in short circuit current $\left(J_{s c}\right)$, open circuit voltage $\left(V_{\mathrm{oc}}\right)$, fill factor (FF), efficiency $(\eta)$ and $\mathrm{EQE}$ was observed. We attribute some slight mismatches of solar cell performance to fabrication process variations. The fact that no performance degradation is observed on the cell fabricated on the reused substrate demonstrates the feasibility of direct reuse of GaAs substrates by applying phosphide base sacrificial layer and the use of concentrated $\mathrm{HCl}$ as the etchant.

Surface tension-assisted ELO process as a new lift-off method. Finally, one major throughput bottleneck in conventional ELO processes is the slow lateral etch rate of the sacrificial layer. Generally speaking, the lateral etch rate of the sacrificial layer is determined by two consecutive processes: the diffusion of the etchant from the solution to the etching front and the reaction of etchant with the sacrificial layer. The etch rate is initially limited by the chemical reaction until the diffusion distance of the etchant causes the etch rate to be limited by the diffusion rate. Performing the ELO process in the chemical reaction rate limited regime is preferred as it can maximize the overall etch rate. In order to do so, several methods with sophisticated setups, for example, weight-assisted $\mathrm{ELO}^{30}$ and etching assisted by roller $^{15,31}$, were developed to accelerate the ELO process. However, these are only single-wafer solutions with consequent low throughput.

To enhance our ELO process throughput, we developed a new method called 'surface tension-assisted (STA) ELO' and its concept is shown in Fig. 4(a). The wafer coated with the photoresist is placed obliquely with an angle $(\theta)$ of $1^{\circ}-20^{\circ}$ from the solution surface, and $\mathrm{HCl}$ is added to the level of the etching front. The wafer surface is protected by the photoresist from being attacked by the acid. As discussed above, the GaAs surface becomes hydrophobic in $\mathrm{HCl}$, so the film floats on the surface of the $\mathrm{HCl}$ solution after lift-off. During the STA-ELO process, surface tension pulls the film away from the substrate and flattens the film on the surface. In the meantime, $\mathrm{HCl}$ replenishes the space between the lifted-off film and the substrate via the capillary 

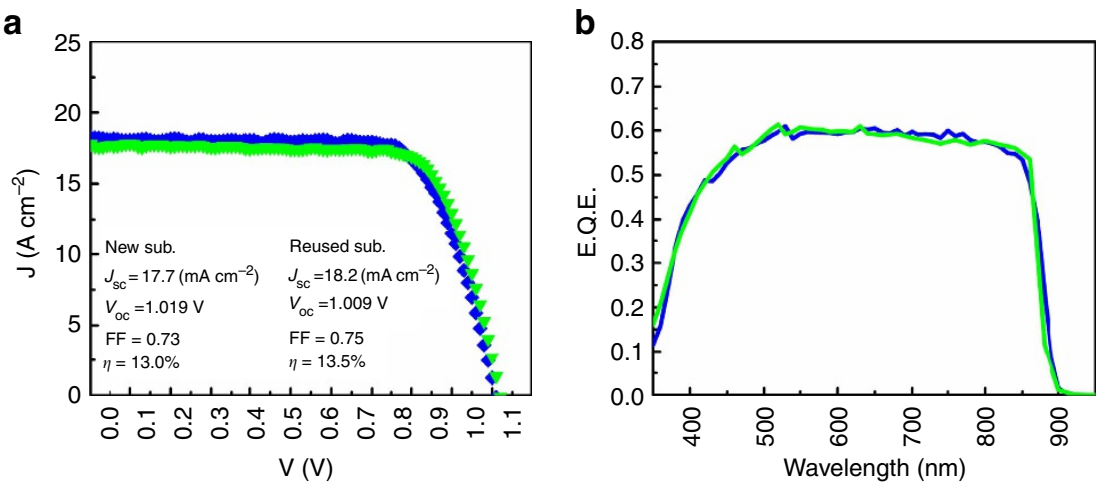

Figure 3 | Performance of single junction GaAs solar cells fabricated on new and reused substrates. (a) Current density versus voltage (J-V) characteristics of GaAs SJ solar cells grown and fabricated on new (green symbols) and reused (blue symbols) substrates. Inset: solar cell performance parameters. (b) EQE of solar cells grown on new (green) and reused (blue) substrates as a function of wavelength. No anti-reflection coating (ARC) was applied.

a
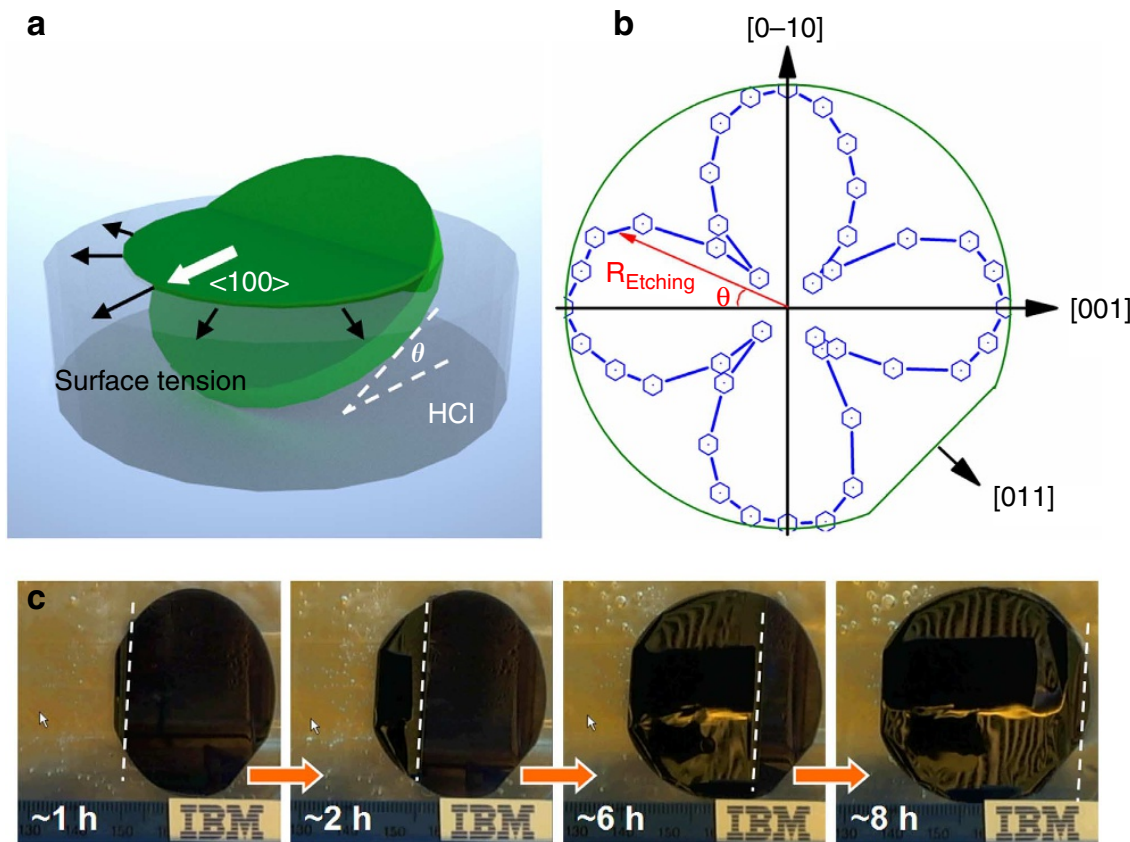

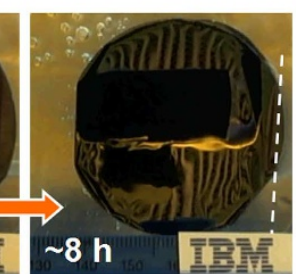

Figure 4 | Surface tension-assisted ELO process. (a) Schematic illustration of the surface tension-assisted (STA) ELO process. (b) Etching rate of InAIP in $\mathrm{HCl}$ as the function of crystallographic direction. The maximum etching rate locates at $\langle 100>$. All the data were normalized by maximum etching rate. (c) Recorded images of the lifted-off film during STA-ELO process. The minor image distortion was caused by the vibration of hood. The InAIP sacrificial layer thickness is $100 \mathrm{~nm}$. The temperature of $\mathrm{HCl}$ solution was kept at $60^{\circ} \mathrm{C}$ and the process took about $8 \mathrm{~h}$. The average lateral etching rate is $\sim 5.9 \mathrm{~mm} \mathrm{~h}{ }^{-1}$ in this demonstration. The etching rate could be increased by further optimization of the process, structure and setup.

force, which causes the process to continue to be reaction limited. Figure 4(b) shows the relationship between the normalized etch rate of the phosphide sacrificial layer and the crystallographic direction of the wafers. The study reveals a fourfold symmetry where the maximum etch rate occurs along the $<100\rangle$ axis, as the etching fronts are terminated by $\{111\} \mathrm{A}^{\text {faces }}{ }^{32}$. As a result, the wafer needs to be tilted along the $<100\rangle$ axis for the highest etch rate and to keep the etching front along the $<100>$ direction. This allows the surface tension of the solution to apply a uniform force on the lifted-off film. The complete STA-ELO process is shown in Fig. 4(c) and in the Supplementary Video S1. The camera reflection on the lifted-off film demonstrates how the surface tension of the solution results in a flat surface. The liftedoff film can be transferred onto almost any substrate for various applications, including either a rigid (Fig. 5(a)) or a flexible substrate (Fig. 5(b)). After the ELO process, the parent wafer only needs to be rinsed thoroughly and then cleaned with a standard wafer cleaning procedure before the next epitaxial growth.

\section{Discussion}

After the STA-ELO process, the GaAs thin film can be transferred onto any thermal-expansion-coefficient (TCE) matched substrates (for example, soda-lime glass) without using any metal supporting layer during ELO process. This helps keep the GaAs surface pristine and the newly transferred film compatible with epitaxy systems for subsequent epitaxial growth. Another advantage is that devices can be fabricated on the GaAs surface first before the film containing the devices is released from the parent substrate. By these means, the device yield can be largely improved, as it reduces the constraint on the highest process temperature allowable imposed by the thermal mismatch between 
the film and the carrier substrate. Figure 6 demonstrates some conceptual devices fully fabricated in advance before our STAELO process: (a) a light-emitting diode (LED) transferred onto Si
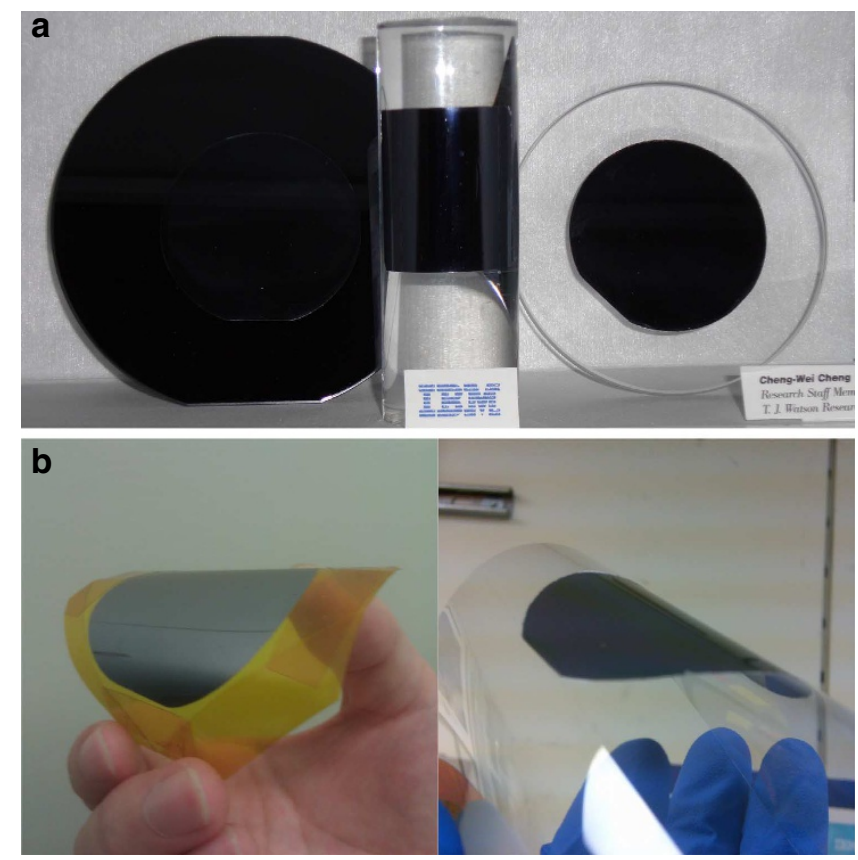

Figure 5 | GaAs thin films transferred to rigid and flexible substrates.

(a) Demonstrations of the transferred GaAs thin films to the rigid Substrate (left, GaAs on 4" Si wafer. Center, GaAs on curved solid object. Right, GaAs on glass) and (b) flexible substrates (left, GaAs on tape. Right, GaAs on flexible sheet). The thickness of the GaAs ranges between 1-3 $\mu \mathrm{m}$ and the diameter of the GaAs is 2" except the film transferred to the curved solid object. substrate (Supplementary Fig. S5), and (b) metal-oxide-semiconductor capacitors (MOSCAP) transferred onto flexible tape. Red light emission in Fig. 6(a) suggests that the integrity of the LED was preserved after the film transfer. Figure 6(b) shows capacitance-voltage measurements from GaAs MOSCAPs at different frequencies, demonstrating no observable difference between devices measured before and after the thin film transfer.

In conclusion, the direct reuse of GaAs substrates has been demonstrated by a newly developed ELO process. Residue-free and atomically flat post-ELO surfaces, resulting from the use of phosphide base sacrificial layers and $\mathrm{HCl}$-based etchants, results in high quality regrown GaAs thin films and enables the regrowth of solar cell structures without observable performance degradation. Furthermore, STA-ELO, a high-throughput lift-off technique, is developed and employed to transfer GaAs thin films onto both rigid and flexible substrates. This technique opens up a suite of potential applications using III-V materials.

\section{Methods}

Material growth. III-V growth, including both arsenide and phosphide base materials, was performed in Thomas Swan close coupled showerhead cold-wall MOCVD system. High-purity $\mathrm{N}_{2}$ was employed as the carrier gas, and the substrate temperature and the reactor pressure were kept at $650^{\circ} \mathrm{C}$ and $100 \mathrm{Torr}$ during the growth. Epilayers were grown on either $2^{\prime \prime} n$-type (Si doped) or p-type (Zn doped) epi-ready GaAs wafers depending on the device structures. Trimethylgallium (TMG), Trimethylaluminum (TMA) and Trimethylindium (TMI) were used as element III precursors. Arsine $\left(\mathrm{AsH}_{3}\right)$ and Phosphine $\left(\mathrm{PH}_{3}\right)$ were used as element $\mathrm{V}$ sources for growing arsenide $(\mathrm{V} / \mathrm{III}=23)$ and phosphide $(\mathrm{V} /$ $\mathrm{III}=400)$ materials, respectively. DiSilane $\left(\mathrm{Si}_{2} \mathrm{H}_{6}\right)$ provided $\mathrm{n}$-type dopants and dimethylzinc was used for p-type doping.

Experiment, device fabrication and characterization. The layers of III-V solar cells were grown on a $2^{\prime \prime} \mathrm{N}$-type GaAs wafers. The wafers were then cut into two pieces with one piece serving as a reference. The other piece went through ELO process to separate the solar cell structure from the substrate, and it was then reloaded into MOCVD system and the same layer structure was regrown. After the regrowth, these two pieces were processed in parallel to complete the final solar cell a

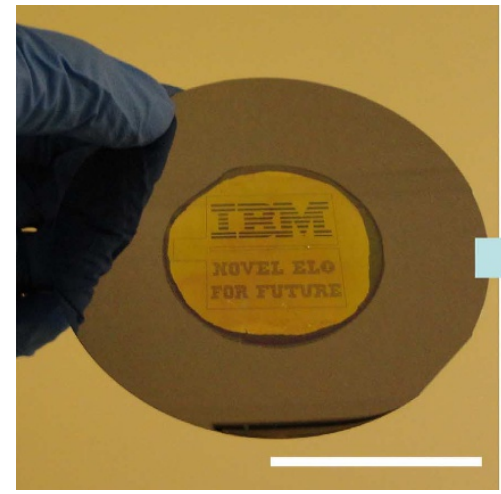

b

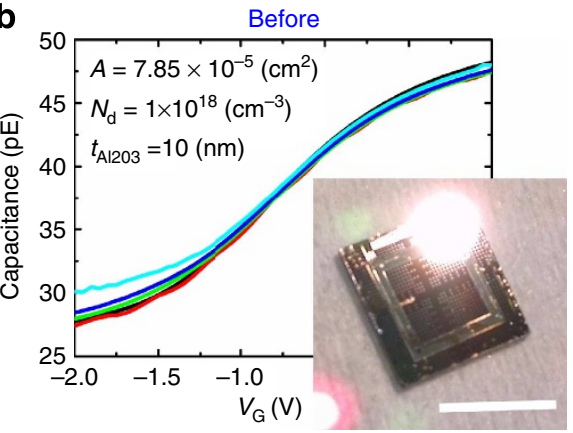

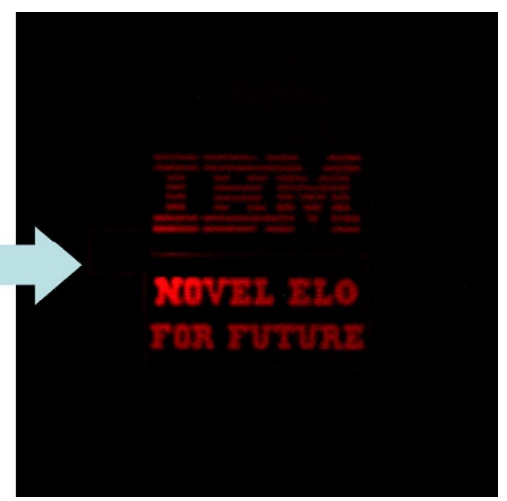

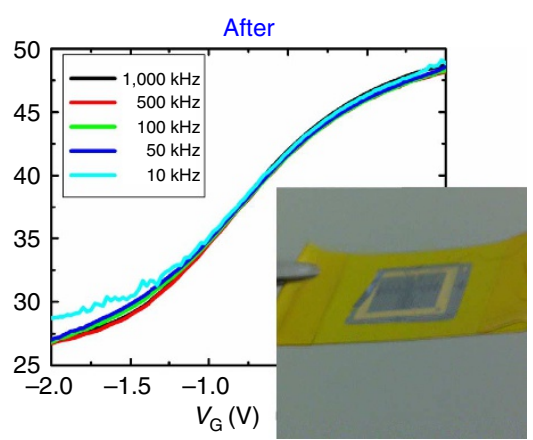

Figure 6 | Demonstration of transferred devices via novel ELO process. (a) Transferred 2" AIGaAs LED on 2" Si wafer and the optical image of light emission. Scale bar, $5 \mathrm{~cm}$. (b) Capacitance-Voltage ( $C-V)$ characteristics of $n-G a A s ~ M O S C A P$ before and after being transferred to a flexible tape. Inset: the optical image of MOSCAPs on a mother substrate and on a flexible tape. Scale bar, $1 \mathrm{~cm}$. 
structures, which is shown in Supplementary Fig. S6. A detailed process flow is described below.

$1 \times 1 \mathrm{~cm}^{2}$ mesa was defined by photolithography and etched to the bottom contact layer by $\mathrm{NH}_{4} \mathrm{OH} / \mathrm{H}_{2} \mathrm{O}_{2} / \mathrm{H}_{2} \mathrm{O}$. The bottom $\mathrm{n}$-ohmic contact was made with thermal evaporated $\mathrm{AuGe} / \mathrm{Ni} / \mathrm{Au}$ and a lift-off process, followed by rapid-thermal annealing (RTA) at $450^{\circ} \mathrm{C}$ for $1 \mathrm{~min}$ in $\mathrm{N}_{2}$ ambient. Then, a shadow mask was applied to define the top p-ohmic contact region by depositing $\mathrm{Au} / \mathrm{Ni}$ and $1 \mu \mathrm{m}$ thick Ag to lower the series resistance. After removing the shadow mask, the top contact layer (p-GaAs) was selectively etched by citric acid/ $\mathrm{H}_{2} \mathrm{O}_{2}$. No RTA was performed for $\mathrm{p}$-type ohmic contact and no anti-reflection coating was applied on the solar cells. The J-V characteristics of GaAs SJ solar cells were measured under simulated AM1.5 solar spectrum at 1 sun intensity.

A similar process flow for the solar cell was applied for LED device fabrication, and the layer structure as well as the final device structure of LED are shown in Supplementary Fig. S7.

MOSCAPs were fabricated by the following process flow. After surface degreasing and ammonia-base native oxide etching, the sample was transferred to an ALD reactor and $10 \mathrm{~nm}$ thick $\mathrm{Al}_{2} \mathrm{O}_{3}$ was deposited at a substrate temperature of $250{ }^{\circ} \mathrm{C}$, followed by $600{ }^{\circ} \mathrm{C}$ post-deposition annealing for $15 \mathrm{~s}$. The substrate contact was defined by photolithography and $\mathrm{Al}_{2} \mathrm{O}_{3}$ was removed by buffered oxide etch (BOE) solution and $\mathrm{n}$-ohmic contact was made by thermal evaporated AuGe/Ni/ $\mathrm{Au}$ and a lift-off process, followed by $\mathrm{N}_{2} \mathrm{RTA}$ at $450^{\circ} \mathrm{C}$ for 1 min. Top Al electrodes of $100 \mu \mathrm{m}$ in diameter were deposited by thermal evaporation through a shadow mask. Finally, the fabricated MOSCAPs were transferred onto a piece of flexible tape. $\mathrm{C}-\mathrm{V}$ characteristics of MOSCAP were measured using Agilent $4284 \mathrm{~A}$ precision LCR metre at frequencies from $1 \mathrm{MHz}$ to $10 \mathrm{kHz}$. The layer structure and the final MOSCAP structure are shown in Supplementary Fig. S8.

\section{References}

1. Bett, A. W., Dimroth, F., Stollwerck, G. \& Sulima, O. V. III-V compounds for solar cell applications. Appl. Phys. A 69, 119-129 (1999).

2. Bosi, M. \& Pelosi, C. The potential of III-V semiconductors as terrestrial photovoltaic devices. Prog. Photovolt. 15, 51-68 (2007).

3. Schwierz, F. \& Liou, J. J. RF transistors: recent developments and roadmap toward terahertz applications. Solid-State Electron 51, 1079-1091 (2007).

4. Chang, K. Y. \& Kai, F. GaAs High-Speed Devices (Wiley \& Sons, 1994).

5. Shockley, W. \& Queisser, H. J. Detailed balance limit of efficiency of p-n junction solar cell. J. Appl. Phys. 32, 510-519 (1961).

6. Konagai, M., Sugimoto, M. \& Takahashi, K. High efficiency GaAs thin film solar cells by peeled film technology. J. Cryst. Growth 45, 277-280 (1978).

7. Bauhuis, G. J., Mulder, P., Haverkamp, E. J., Huijben, J. C. C. M. \& Schermer, J. J. 26.1\% thin-film GaAs solar cell using epitaxiallift-off. Solar Energy Materials Solar Cells 93, 1488-1491 (2009).

8. Jongseung, Yoon et al. Rogers GaAs photovoltaics and optoelectronics using releasable multilayer epitaxial assemblies. Nature 465, 329-333 (2010).

9. Bauhuis, G. J. et al. Wafer reuse for repeated growth of III-V solar cells. Prog. Photovolt Res. Appl. 18, 155-159 (2010)

10. Voncken, M. M. A. J. et al. Etching AlAs with HF for epitaxial lift-off applications. J. Electrochem. Soc. 151, G347-G352 (2004).

11. Clawson, A. R. Guide to reference on III-V semiconductor chemical etching. Mater. Sci. Eng. A 31, 1-438 (2001).

12. Flemish, J. R. \& Jones, K. A. Selective wet etching of GaInP,GaAs and InP in solutions of $\mathrm{HCl}, \mathrm{CH}_{3} \mathrm{COOH}$, and $\mathrm{H}_{2} \mathrm{O}_{2}$. J. Electrochem. Soc. 140, 844-847 (1993).

13. Hjort, K. Sacrificial etching of III-V compounds for micromechanical devices. J. Micromech. Microeng. 6, 370-375 (1996).

14. Meitl, M., Bower, C., Menard, E., Carter, J., Gray, A. \& Bonafede, S. Materials and processes for releasing printable compound semiconductor devices. International publication number: WO 2012/018997 A2; 2012.

15. Schermer, J. J. et al. Epitaxial Lift-Off for large area thin film III/V devices. Phys. Stat. Sol. 202, 501-508 (2005).

16. Niftrik, A. T. J., van, Schermer, J. J., Bauhuis, G. J., Mulder, P., Larsen, P. K. \& Kelly, J. J. A diffusion and reaction related model of the epitaxial lift-off process. J. Electrochem. Soc. 154, D629-D635 (2007).

17. Niftrik, A. T. J. et al. HF species and dissolved oxygen on the epitaxial lift-off process of gaas using alasp release layers. J. Electrochem. Soc. 155, D35-D39 (2008).
18. Lide, D. R. CRC Handbook of Chemistry and Physics 87th edn (Taylor \& Francis, 2006-2007).

19. Lee, K., Zimmerman, J. D., Xiao, X., Sun, K. \& Forrest, S. R. Reuse of GaAs substrate for epitaxial lift-off by employing protection layers. J. Appl. Phys. 111, 033527 (2012).

20. Notten, P. H. L. The etching of InP in $\mathrm{HCl}$ solutions: a chemical mechanism. J. Electrochem. Soc. 131, 2641-2644 (1984).

21. Lee, J. W., Pearton, S. J. \& Abernathy, C. R. Wet chemical etching of $\mathrm{Al}_{0.5} \mathrm{In}_{0.5} \mathrm{P}$. J. Electrochem. Soc. 142, L100-L102 (1995).

22. Lothian, J. R., Kuo, J. M., Ren, F. \& Pearton, S. J. Plasma and wet chemical etching of InGaP. J. Electron. Mater. 21, 441-445 (1992).

23. Neves, S. das \& Paoli, M.-A. de A quantitative study of chemical etching of InP. J. Electrochem. Soc. 140, 2599-2603 (1993).

24. Bandaru, P. \& Yablonovitch, E. Semiconductor surface-molecule interactions wet etching of InP by $\alpha$-hydroxy acids. J. Electrochem. Soc. 149, G599-G602 (2002).

25. Osakabe, S. \& Adachi, S. Study of GaAs (100) surfaces treated in aqueous $\mathrm{HCl}$ solutions. J. Jpn. Appl. Phys. 36, 7119-7125 (1997).

26. Matsushita, K., Miyazaki, S., Okuyama, S. \& Kumagai, Y. Observation of HCland HF- treated GaAs surfaces by measuring contact angles of water droplets. J. Jpn. Appl. Phys. 33, 4576-4580 (1994).

27. Ishikawa, Y., Ishii, H., Hasegawa, H. \& Fukui, T. Macroscopic electronic behavior and atomic arrangements of GaAs surfaces immersed in $\mathrm{HCl}$ solution. J. Vac. Sci. Technol. B 12, 2713-2719 (1994).

28. Song, Z., Shogen, S., Kawasaki, M. \& Suemune, I. X-ray photoelectron spectroscopy and atomic force microscopy surface study of $\mathrm{GaAs}(100)$ cleaning procedures. J. Vac. Sci. Technol. B 13, 77-82 (1995).

29. Lu, Z. H. et al. Passivation of $\mathrm{GaAs}(111) \mathrm{A}$ surface by $\mathrm{Cl}$ termination. Appl. Phys. Lett. 67, 670-672 (1995).

30. Schermer, J. J. et al. Appl. Phys. Lett. 76, 2131-2133 (2000).

31. Shiu, K. T., Zimmerman, J., Wang, H. \& Forrest, S. R. Ultrathin film, high specific power InP solar cells on flexible plastic substrates. Appl. Phys. Lett. 95, 223503-223505 (2009)

32. Cich, M. J., Johnson, J. A., Peake, G. M. \& Spahn, O. B. Crystallographic dependence of the lateral undercut wet etching rate of InGaP in HCl. Appl. Phys. Lett. 82, 651-653 (2003).

\section{Acknowledgements}

The authors thank Dr Tze-Chiang Chen, Dr Ghavam Shahidi, Dr Harold Hovel, Dr Joel P. de Souza, and Dr Hsin-yu Tsai for all the supports and professional discussions and suggestions in this work in IBM Watson Research Centre. The authors also thank Dr Yang Li, Dr Nan Yang, Adam Jandl, Kunal Mukherjee, Ryan Iutzi, Tim Milakovich and Prithu Sharma for helps in III-V materials growths at MIT.

\section{Author contributions}

C.-W.C. designed and executed the study and wrote the manuscript. K.-T.S. assisted C.-W.C. to execute all the experiments. N.L. performed the AFM measurements. The images were taken with assistance from N.L. S.-J.H. and L.S. contributed to the developments of the thin film transfer process. S.-J.H. made figures and contributed to the manuscript preparation. D.K.S. supervised the project. All the authors joined the discussion and provided comments.

\section{Additional information}

Supplementary Information accompanies this paper at http://www.nature.com/ naturecommunications

Competing financial interests: The authors declare no competing financial interests.

Reprints and permission information is available online at http://npg.nature.com/ reprintsandpermissions/

How to cite this article: Cheng, C.-W. et al. Epitaxial lift-off process for gallium arsenide substrate reuse and flexible electronics. Nat. Commun. 4:1577 doi: 10.1038/ncomms2583 (2013). 